\title{
A Database Approach to Symbolic Music Content Management
}

\author{
Zoé Faget $^{1,3}$, Philippe Rigaux ${ }^{1,2}$ \\ ${ }^{1}$ Lamsade, Univ. Paris-Dauphine, ${ }^{2}$ WebDam, INRIA-Saclay, ${ }^{3}$ Armadillo.
}

\begin{abstract}
The paper addresses the problem of content-based access to large repositories of digitized music scores. We propose a data model and query language that allow an in-depth management of musical content. In order to cope with the flexibility of music material, the language is designed to easily incorporate user-defined functions at early steps of the query evaluation process. We describe our architectural vision, develop a formal description of the language, and illustrate a user-friendly syntax with several classical examples of symbolic music information retrieval.
\end{abstract}

\section{Introduction}

Most of the proposals devoted so far to analysis methods or similarity searches on symbolic music focus on the accuracy and/or relevancy of the result, and implicitly assume that these procedures apply to a single score, or to a small collection $[2,3,13,16]$. While useful, this approach gives rise to several issues when the collection consists of thousands of scores, with heterogeneous descriptions.

A first issue is related to software engineering and architectural concerns. A large score repository - say, a score digital library - provides search, extraction and transformation services to many differents users or applications. Consistency, reliability, and security concerns call for the definition of a single consistent data management interface for these services. In particular, one can hardly envisage the publication of ad-hoc search procedures that merely exhibit the bunch of methods and algorithms developed for each specific retrieval task. The multiplication of these services would quickly overwhelm external users. Worse, the combination of these functions, which is typically a difficult matter, would be left to external applications. In complex systems, the ability to compose fluently the data manipulation operators is a key to both expressive power and computational efficiency.

Therefore, on-line communities dealing with scores and/or musical content are often limited either by the size of their corpus or the range of possible operations, with only one publicized strong feature. Examples of on-line communities include Mutopia [26], MelodicMatch [27] or Musipedia [28]. Wikifonia [29] offers a wider range of services, allowing register users to publish and edit sheet music. One can also cite the OMRSYS platform described in [8].

A second issue pertains to scalability. With the ongoing progress in digitization, optical recognition and user content generation, we must be ready to 
face an important growth of the volume of music content that must be handled by institutions, libraries, or publishers. Optimizing accesses to large datasets is a delicate matter which involves many technical aspects that embrace physical storage, indexing and algorithmic strategies. Such techniques are usually supported by a specialized data management system which releases applications from the burden of low-level and intricate implementation concerns.

To our knowledge no such system exists at this moment for the domain of symbolic music management. The HumDrum toolkit is a widely used automated musicological analysis tool [14,22], but representation remains at a low level. A HumDrum based system will lack in flexibility and will depend too much on how files are stored. This makes difficult the development of indexing of optimization techniques. Another possible approach would be a system based on MusicXML, an XML based file format $[12,24]$. It has been suggested recently that XQuery may be used over MusicXML for music queries [11], but XQuery is a generalpurpose query language which hardly adapts to the specifics of symbolic music manipulation.

Our goal here is not to propose a new data format, given the great variety of formats already present, but rather to present a data model specifically designed for symbolic music management. Our objective in this paper is to lay the ground for a score management system by proposing one of its core components, namely a logical data model and its associated query language. Our approach is based on the idea that the management of structured scores corresponds, at the core level, to a limited set of fundamental operations that can be defined and implemented once for all. We also take into account the fact that the wide range of user needs calls for the ability to associate these operations with user-defined functions at early steps of the query evaluation process. Modeling the invariant operators and combining them with user-defined operations is the main goal of our design effort. Among numerous advantages, this allows the definition of a stable and robust query() service which does not need ad-hoc extensions as new requirements arrive.

We do not claim (yet) that our model and upcoming implementation will scale easily, but a high level representation like our model is a pre-requisit in order to allow the necessary flexibility for such futur optimization.

The language offers a generic mechanism to search and transform music notation. Its implementation is currently in progress and we plan to experiment its use in the Neuma Digital Score Library [30], devoted to large collections of monodic and polyphonic music from the French Modern Era $\left(16^{t h}-18^{\text {th }}\right.$ centuries).

The rest of this paper first discusses related work (Section 2). Section 3 presents the motivation and the context of our work. Section 4 then exposes the formal fundations of our model. A user query language is introduced with examples in Section 5. Section 6 concludes the paper. 


\section{Related work}

The past decade has witnessed a growing interest in techniques for representing, indexing and searching (by content) music documents. The domain is commonly termed "Music Information Retrieval" (MIR) although it covers many aspects beyond the mere process of retrieving documents. We refer the reader to [19] for an introduction. Systems can manipulate music either as audio files or in symbolic form. The symbolic representation offers a structured representation which is well suited for content-based accesses, sophisticated manipulations, and analysis [13].

An early attempt to represent scores as structured files and to develop search and analysis functions is the HumDrum format. Both the representation and the procedures are low-level (text files, Unix commands) which make them difficult to integrate in complex application. Recent works try to overcome these limitations $[22,16]$. Musipedia proposes several kinds of interfaces are to search the database by content. MelodicMatch is a similar software analysing music through pattern recognition, enabling search for musical phrases in one or more pieces. MelodicMatch can search for melodies, rhythms and lyrics in MusicXML files.

The computation of similarity between music fragments is a central issue in MIR systems [10]. Most proposal focus on comparisons of the melodic profiles. Because music is subject to many small variations, approximate search is of order, and the problem is actually that of finding nearest neighbors to a given pattern. Many techniques have been experimented, that vary depending on the melodic encoding and the similarity measure. See $[9,4,1,7]$ for some recent proposals. The Dynamic Time Warping (DTW) distance is a well-known popular measure in speech recognition $[21,20]$. It allows the non-linear mapping of one signal to another by minimizing the distance between the two. The DTW distance is usually chosen over the less flexible Euclidian distance for time series alignment [5]. The DTW computation is rather slow but recent works show that it can be efficiently indexed $[25,15]$.

We are not aware of any general approach to model and query music notation. A possible approach would be to use XQuery over MusicXML documents as suggested in [11]. XQuery is a general-purpose query language, and its use for music scores yields complicated expressions, and hardly adapts to the specifics of the objects representation (e.g., temporal sequences). We believe that a dedicated language is both more natural and more efficient. The temporal function approach outlined here can be related to time series management [17].

\section{Architecture}

Figure 1 outlines the main components of a Score Management System built around our data model. Basically, the architecture is that of a standard DBMS, and we actually position our design as an extension of the relational model, making it possible to incorporate the new features as components of an extensible relational system. The model consists of a logical view of score content, along 


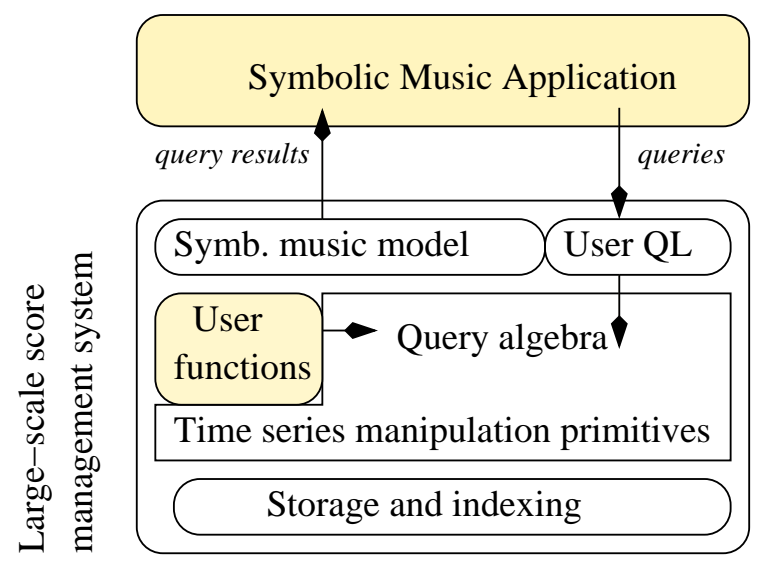

Fig. 1. Approach overview

with a user query language and an algebra that operates in closed form (i.e., each operator consumes and produces instances of the model). The algebra can be closely associated with a library of user-defined functions which must be provided by the application and allow to tailor the query language to a specific domain. Functions in such a library must comply to constraints that will be developed in the next section.

This approach brings, to the design and implementation of applications that deal with symbolic music, the standard and well-known advantages of specialized data management systems. Let us just mention the few most important: (i) ability to rely on a stable, well-defined and expressive data model, (ii) independence between logical modeling and physical design, saving the need to confront programmers with intricate optimization issues at the application level and (iii) efficiency of set-based operators and indexes provided by the data system.

Regarding the design of our model, the basic idea is to extend the relational approach with function-valued attributes. Each such attribute represents a (peculiar) temporal function that maps a discrete temporal space to values in some domain.

The model supports the representation of polyphonic pieces composed of "voices", each voice being a sequence of "events" in some music-related domain (notes, rests, chords) such that only one event occurs at a given instant. Adding new domains allows to extend the concept to any sequence of symbols taken from a finite alphabet. This covers monodies, text (where the alphabet consists of syllables) as well as, potentially, any sequence of music-related information (e.g., fingerings, performance indications, etc.).

We model a musical piece as Synchronized Time Series (STS). Generally speaking, a time series is an ordered sequence of values taken at equal time intervals. Some of the traditional domains that explicitly produce and exploit time series are, among others, sales forecasting, stock market analysis, process 
and quality control, etc. [6]. In the case of music notation, "time" is not to be understood as a classic calendar (i.e., days or timestamps) as in the previously mentioned examples but rather, at a more abstract level, as a sequence of events where the time interval is the smallest note duration in the musical piece.

Consider now a digital library that stores music information and provides query services on music collections. A music piece consists of one or several parts which can be modelled as time series, each represented by a score. Fig. 2 is a simple example of a monodic piece, whereas polyphonic music pieces (Fig. 3) exhibit a synchronization of several parts.

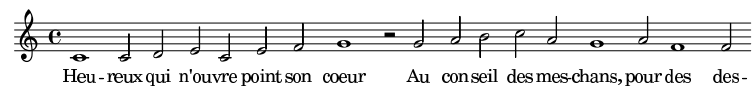

Fig. 2. A monodic score

The temporal domain of interest here is an abstract representation if the temporal features of the times series. Essentially, these features are (i) the order of the musical "events" (notes, rests), (ii) the relative duration of the events, and (iii) the required synchronization of the different parts (here lyrics and notes).

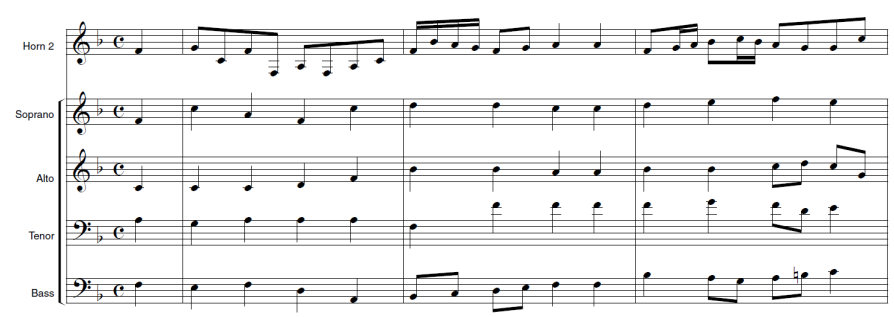

Fig. 3. A polyphonic score

Here are a few examples of queries:

1. Get the scores whose lyrics contains the word 'conseil' (selection)

2. Get the melodic part that corresponds to the word 'conseil' (selection and temporal join)

3. Find a melodic pattern. (search by similarity)

When a collection of musical score is to be studied, queries regarding common features are also of interest. For example

1. Find all musical pieces in the collection starting with the note $C$ and getting to $G$ in 5 times unit. 
2. Find if minor chords occur more often than major chord synchronized with the word 'tot' in Bach's work.

We provide an algebra that operates in closed form over collections of scores. We show that this algebra expresses usual operations (e.g., similarity search), achieves high expressiveness through unbounded composition of operators and natively incorporates the ability to introduce user-defined function in query expressions, in order to meet the needs of a specific approach to symbolic music manipulation. Finally, we introduce a user-friendly query language to express algebraic operations. We believe that our approach defines a sound basis to the implementation of a specialized query language devoted to score collections management at large scale.

\section{The Data Model}

\subsection{Preliminaries}

A musical domain dom $_{\text {music }}$ is a product domain combining heterogenous musical informations. For example, the domain of a simple monodic score is

$$
\operatorname{dom}_{\text {music }}=\operatorname{dom}_{\text {pitch }} \times \operatorname{dom}_{r y t h m} .
$$

Any type of information that can be extracted from symbolic music (such as measures, alterations, lyrics...) can be added to the domain. Each domain contains two distinguished values: the neutral value $T$ and the null value $\perp$. The Boolean operations $\wedge$ (conjunction) and $\vee$ (disjunction) verify, for any $a \in$ dom, $a \wedge \perp=\perp, a \wedge \top=a$ and $a \vee \perp=a \vee \top=a$. In some cases, $\perp$ and $\top$ can also be viewed as false and true.

With a given musical domain comes a set of operations provided by the user and related to this specific domain. When managing a collections of Choir parts, a function such as $\max ($ ) which computes the highest pitch is meaningful, and becomes irrelevant when managing of collection of Led Zeppelin tablatures. Basic functions to single out each part of the complex music domain are implicit (rythm, pitch, lyrics etc...).

We subdivise the time domain $\mathcal{T}$ into a defined, regular, repeated pattern. The subdivision of time is the smallest interval between two musical events. The time domain $\mathcal{T}$ is then a countable ordered set isomorphic to $\mathbb{N}$. We introduce a set of internal time functions, designed to operate on the time domain $\mathcal{T}$. We define as $\mathcal{L}$ the class of functions from $\mathcal{T}$ to $\mathcal{T}$ otherwise known as internal time functions (ITF). An important sub-class of $\mathcal{L}$ is the set of linear functions of the form $t \rightarrow \alpha t+\beta$. We denote them temporal scaling functions in what follows, and further distinguish the families of warping functions of the form $\operatorname{warp}^{\alpha}: \mathcal{T} \rightarrow \mathcal{T}$, $t \mapsto \alpha t$ and shifting functions shift $t^{\beta} \mathcal{T} \rightarrow \mathcal{T}, t \mapsto t+\beta$.

A musical time series (or voice) is a mapping from a time domain $\mathcal{T}$ into a musical domain $\mathbf{d o m}_{m u s i c}$. When dealing with a collection of scores sharing a number of common properties, we introduce the schema of a relation. The 
schema distinguishes atomic attributes names (score_id, author, year...) and times series (or voices) names. We denote by TS([dom]) the type of these attributes, where [dom] is the domain of interest. Here is, for example, the schema of a music score

Score(Id : int, Composer : string, Voice : TS(vocal), Piano : TS(polyMusic)).

Note that the time domain is shared by the vocal part and the piano part. The domain vocal adds the lyrics domain to the classic music domain:

$$
\mathbf{d o m}_{\text {vocals }}=\operatorname{dom}_{\text {pitch }} \times \operatorname{dom}_{\text {rythm }} \times \operatorname{dom}_{\text {lyrics }} .
$$

The domain polymusic is the product

$$
\mathbf{d o m}_{\text {polymusic }}=\left(\mathbf{d o m}_{\text {pitch }} \times \operatorname{dom}_{\text {rythm }}\right)^{N} .
$$

We will now define two sets of operators gathered into two algebras: the (extended) relational algebra, and the times series algebra.

\subsection{The relational algebra $\mathrm{ALG}_{(R)}$}

The $\mathrm{ALG}_{(R)}$ algebra consists of the usual operators selection $\sigma$, product $\times$, union $\cup$ and difference - , along with an extended projection $\Pi$. We present simple examples of those operators.

Selection, $\boldsymbol{\sigma}$. Select all scores composed by Louis Couperin:

$$
\sigma_{\text {author }{ }^{\prime} \text { Louis Couperin' }}(\text { Score })
$$

Projection, $\boldsymbol{\pi}$. We want the vocal parts from the Score schema without the piano part. We project the piano out:

$$
\pi_{\text {vocals }}(\text { Score })
$$

Product, $\times$. Consider a collection of duets, split into the individual vocal parts of male and female singers, with the following schemas

$$
\begin{gathered}
\text { Male_Part }(I d: \text { int, Voice }: \text { TS(vocals)), } \\
\text { Female_Part }(I d: \text { int, Voice }: \text { TS(vocals)). }
\end{gathered}
$$

To get the duet scores, we cross product a female part and a male part. We get the relation

$$
\text { Duet(Id: int, Male_V : TS(vocals), Female_V: TS(vocals)). }
$$

Note that the time domain is implicitly shared. In itself, the product doesn't have much interest, but together with the selection operator it becomes the join operators $\bowtie$. In the previous example, we shouldn't blindly associate any male and female vocal parts, but only the ones sharing the same Id.

$$
\sigma_{\text {M.Id }=\text { F.Id }}(\text { Male } \times \text { Female }) \equiv \text { Male } \bowtie_{I d=I d} \text { Female } .
$$


Union $\cup$. We want to join the two consecutive movements of a piece which have two separate score instances.

$$
\text { Score }=\text { Score }_{p t 1} \cup \text { Score }_{p t 2} .
$$

Beyond classical relational operators, we introduce an emptyness test $\emptyset$ ? that operates on voices and is modeled as: $\emptyset^{?}(s)=$ false if $\forall t, s(t)=\perp$, else $\emptyset^{?}(s)=$ true. The emptynes test can be introduced in selection formulas of the $\sigma$ operator.

Consider once more the relation Score. We want to select all scores featuring the word 'Ave' in the lyrics part of $V$. We need a user function $m:($ lyrics $) \rightarrow$ $(\perp, \top)$ such that $m\left({ }^{\prime} A v e^{\prime}\right)=\top$, else $\perp$. Lyrics not containing 'Ave' are transformed into the "empty" time series $t \mapsto \perp, \forall t$. The algebraic expression is:

$$
\sigma_{\emptyset ?(W)}\left(\Pi_{[I d, V, W: m(\operatorname{lyrics}(V))]}(\text { Score })\right) .
$$

\subsection{The time series algebra $\mathrm{Alg}_{(T S)}$}

We now present the operators of the time series algebra $\operatorname{ALG}_{(T S)}(\circ, \oplus, \mathbf{A})$. Each operator takes one or more time series as input and produces a time series. This way, operating in closed form, operators can be composed. They allow, in particular: alteration of the time domain in order to focus on specific instants (external composition); combination of two times series to form a new one (addition operator); alignment of time series fragments for matching purposes (aggregation).

In what follows, we take an instance $s$ of the Score schema to run several examples.

The external composition o composes a time series $s$ with an internal temporal function $l$. Assume our score $s$ has two movements, and we only want the second one. Let shift $t^{n}$ be an element of the shift family functions parametrized by a constant $n \in \mathbb{N}$. For any $t \in \mathcal{T}, s \circ$ shift $t^{n}(t)=s(t+n)$. In other words, $s \circ$ shift $^{n}$ is the time series extracted from $s$ where the first $n$ events are ignored. We compose $s$ with the shift ${ }^{L}$ function, where $L$ is the length of the first movement, and the resulting time series $s \circ$ shift $t^{L}$ is our result.

Imagine now that we want only the first note of every measure. Assuming we are in $4 / 4$ and the time unit is one fourth, we compose $s$ with warp $^{16}$. The time series $s \circ$ warp $^{16}$ is the time series where only one out of sixteen events are considered, therefore the first note of every measure.

We now give an example of the addition operator $\oplus$. Let $\operatorname{dom}_{\text {pitch }}$ be the domain of all musical notes and dom $_{\text {int }}$ the domain of all musical intervals. We

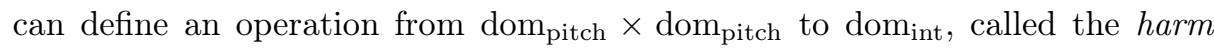
operator, which takes two notes as input and computes the interval between these two notes. Given two time series representing each a vocal part, for instance $V_{1}=$ soprano, $V_{2}=$ alto, we can define the time series

$$
V_{1} \oplus_{h a r m} V_{2}
$$


of the harmonic progression (i.e., the sequence of intervals realized by the juxtaposition of the two voices).

Last, we present the aggregation mechanism A. A typical operation that we cannot yet obtained is the "windowing" which, at each instant, considers a local part of a voice $s$ and derives a value from this restricted view. A canonical example is pattern matching. So far we have no tool allowing us to compare a pattern $P$ with all subsequences of a time series $s$. The intuitive way to do pattern matching is to build all subsequences from $s$ and compare $P$ with each of them, using an appropriate distance. This is what the aggregation mechanism does, in two steps:

1. first, at each instant $\tau$, a TS $s_{\tau}^{\prime}$ is derived from $s$ thanks to a derivation operator $d_{\lambda}$ where $\lambda$ is an internal time function ;

2. second a user aggregation function $\gamma \in \Gamma$ is applied to the TS $s_{\tau}^{\prime}$, yielding an element from dom.

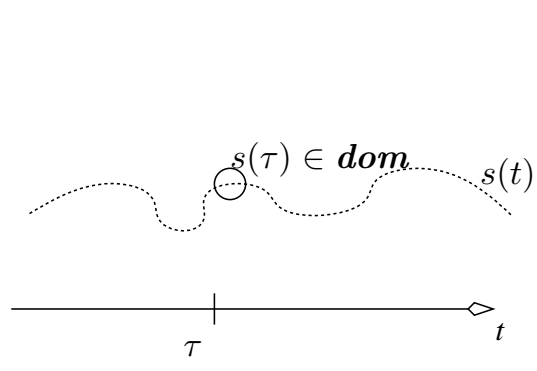

a. Function $s(t)$

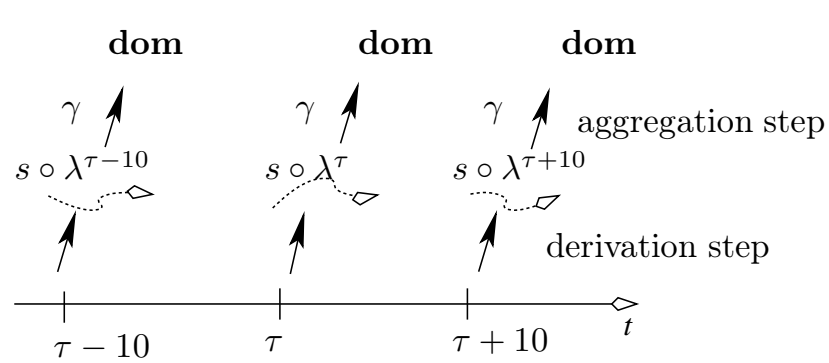

b. Sequence of derived functions $d_{\lambda}(s)$

Fig. 4. The derivation/aggregation mechanism

Fig. 4 illustrates this two-steps process. At each instant $\tau$ a new function is derived. At this point, we obtain a sequence of time series, each locally defined with respect to an instant of the time axis. This sequence corresponds to local views of $s$, possibly warped by temporal functions. Note that this intermediate structure is not covered by our data model.

The second step applies an aggregation function $\gamma$. It takes one (or several, depending on the arity of $\gamma$ ) derived series, and produces an element from dom. The combination of the derivation and aggregation steps results in a time series that complies to the data model.

To illustrate the derivation step, we derive our score $s$ with respect to the shift function :

$$
d_{\text {shift }}(s)=\left(s \circ \text { shift } t^{0}, s \circ \text { shift } t^{1}, \ldots, s \circ \operatorname{shift} t^{n}\right) .
$$

The aggregation step takes the family of time series obtained with the derivation step and applies a user function to all of them. For our ongoing example, 
this translates to applying the user function $D T W_{P}$ (which computes the DTW distance between the input time series $s$ and the pattern $P)$ to $d_{\text {shift }}(s)$. We denote this two steps procedure by the following expression:

$$
\mathbf{A}_{\mathrm{DTW}_{\mathrm{P}}, \operatorname{shift}}(\mathrm{s}) \text {. }
$$

\subsection{Example: Pattern matching using the DTW distance}

To end this section, we give an extended example using operators from $\operatorname{ALG}_{(R)}$ and $\mathrm{Alg}_{(T S)}$. We want to compute, given a pattern $P$ and a score, the instant $t$ where the Dynamic Time Warping (DTW) distance between $P$ and $V$ is less than 5. First, we compute the DTW distance between $P$ and the voice $V_{1}$ of a score, at each instant, thanks to the following $\mathrm{ALG}_{(T S)}$ expression:

$$
e=\mathbf{A}_{\mathrm{dtwP}, \operatorname{shift}}\left(\mathrm{V}_{1}\right)
$$

where $d t w_{P}$ is the DTW function. Expression $e$ defines a time series that gives, at each instant $\alpha$, the DTW distance between $P$ and the sub-series of $V_{1}$ that begins at $\alpha$. A selection (from $\mathrm{ALG}_{(T S)}$ ) keeps the values in $e$ below 5 , all others being set to $\perp$. Let $\psi$ be the formula that expresses this condition. Then:

$$
e^{\prime}=\sigma_{\psi}(e) .
$$

Finally, expression $e^{\prime}$ is applied to all the scores in the Score relation with the $\Pi$ operator. An emptyness test can be used to eliminate those for which the DTW is always higher that 5 (hence, $e^{\prime}$ is empty).

$$
\sigma_{\emptyset ?\left(e^{\prime}\right)}\left(\Pi_{\left[\text {composer }, V_{1}, V_{2}, e^{\prime}\right]}(\text { Score })\right) .
$$

\section{User query language}

Since our model is essentially based on a relational approach, a limited extension of SQL suffices to obtain a user-friendly syntax for our algebraic expressions. Basically, the extension consists of let clauses to define variables as expressions from $\mathrm{ALG}_{(T S)}$, and a construct clause, corresponding to $\Pi$, and extending the standard select. In this section we present first some standard query examples, then move to some more elaborate examples.

\subsection{Basic queries and operations on score}

The first query simply retrieves the songs from Gabriel Fauré (composer), along with their ambitus (minimal and maximal notes in the voice time series).

from Score

construct id, voice, $\min ($ voice), $\max ($ voice)

where composer='Faure' 
In this query, we use $\min ()$ and $\max ()$ which are examples of functions associated to the musical domain (modern query languages, e.g., XQuery, provide this facility). Other examples of such functions include: tonality, genre, mode, whenever such concepts are relevant. Functions can be either aggregation functions (mapping a time series to a value of the domain, e.g., $\min ()$ and $\max ())$ or scalar functions (mapping scalar values to values, e.g. transpose()). The latter can be used to construct new voices, as shown by the following example which transposes the voice one octave up, and keeps only those that do not exceed C5.

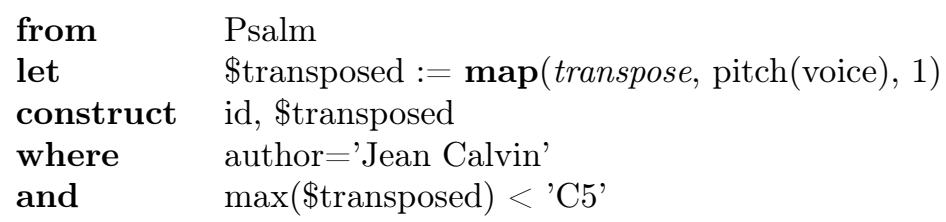

Clauses from and construct (the latter being equivalent to the usual select) are inverted with respect to regular SQL. This permits the introduction of complex expression with a let clause that may refer to the base relations declared in the from. let defines a new voice from existing ones. The following query for instance constructs a "summary" of monodies, taking one note after 4 time units, starting from the third instant.

$\begin{array}{ll}\text { from } & \text { Psalm } \\ \text { let } & \$ \mathrm{t}:=\text { time(voice) } \\ \text { let } & \$ \text { summary }:=\operatorname{voice}(4 * \$ \mathrm{t}+3) \text { where } \$ \mathrm{t} \leq 50 \\ \text { construct } & \text { id, } \$ \text { summary }\end{array}$

In the same spirit, we want to find all the whole notes in a voice.

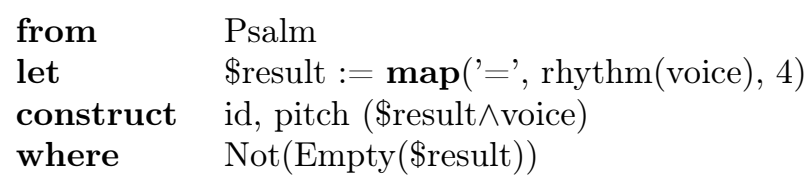

In the mapping process, any voice not containing a whole note is transformed into an "empty" time series and therefore not considered. Here is another example using the emptyness test: we want all scores without alteration.

$\begin{array}{ll}\text { from } & \text { Score } \\ \text { let } & \$ \mathrm{a}:=\text { alteration(voice) } \\ \text { construct } & \text { id, voice } \\ \text { where } & \text { Empty }(\$ a)\end{array}$




\subsection{Pattern matching}

Assume now that we search a pattern in a voice. The following examples shows a simple search of the word 'Heureux' in the lyrics of a voice.

$$
\begin{array}{ll}
\text { from } & \text { Psalm } \\
\text { construct } & \text { id, voice } \\
\text { where } & \text { contains (lyrics(voice), 'Heureux') }
\end{array}
$$

In a very close fashion, a more complete (yet usual) operation searches for the occurrences of a pattern anywhere in a voice, possibly using an approximate search based on a distance function. Assume for instance that we search for a pattern $P$ in a voice, such that the Dynamic Time Warping distance (computed with the $d t w$ function) is less than 5 . Here is the query expression.

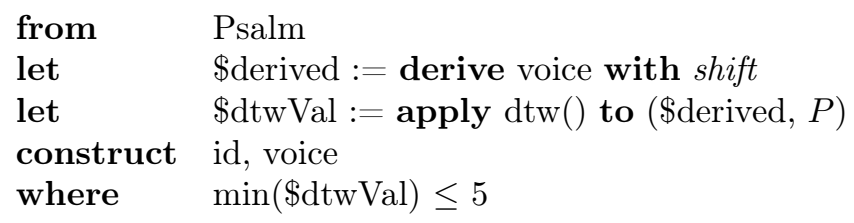

The first let clause "derives" the voice with respect to the shift ITF as explained in Section 4. The second let clause applies the DTW operator to each occurrence of the family of derived functions, thanks to the apply clause. In spirit, this is similar to the combination of the nesting and aggregate operations that underly the group by clause of standard SQL.

One obtains a new voice, named dtwVal, which is itself a time function giving the DTW distance at each instant. The where clause gets rid of those tuples for which the DTW is never less than 5. Note that one could exploit the dtwVal to access precisely to those instants at which the DTW values match a given condition.

An even more elaborate query is to establish all melodic fragments associated to the word "Tot" in Bach's work.

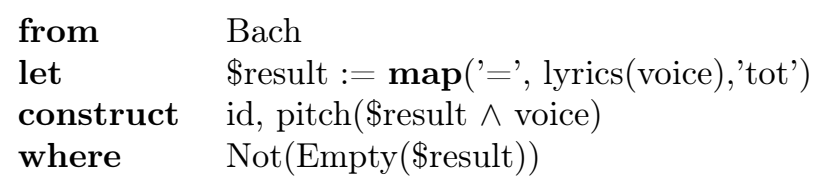

In this example, the notion of synchronization is essential.

\subsection{Application to music analysis}

We want to find all pieces that contain two consecutive fifth between bass and alto parts. The scalar function used here is the harm function which computes the interval between two voices. 


$\begin{array}{ll}\text { from } & \text { Choir } \\ \text { let } & \text { \$harm }:=\operatorname{map}(\text { harm, bass, alto }) \\ \text { let } & \text { \$fifth }:=\operatorname{map}('=, \text { hharm, '3,5') } \\ \text { construct } & \text { id, voice } \\ \text { where } & \text { contains }(\$ \text { fifth, [true, true] })\end{array}$

In the context of music content management where needs differ greatly from one area to another, it is crucial to allow user-defined functions. For our last example, we assume a "segmentation" user function capable of recognizing phrases from structured musical content. Given a voice $V$ as input, phrase $(V)$, produces a new time series taking values in $\{\mathrm{B}, \mathrm{M}, \mathrm{E}\}$ (which stand respectively for Beginning, Middle and End of phrases). We also assume the existence of a user function mode() taking a monodic voice as input and producing the time series of all degrees from the musical range. We want to count the number of phrases that end on the subdominant pitch.

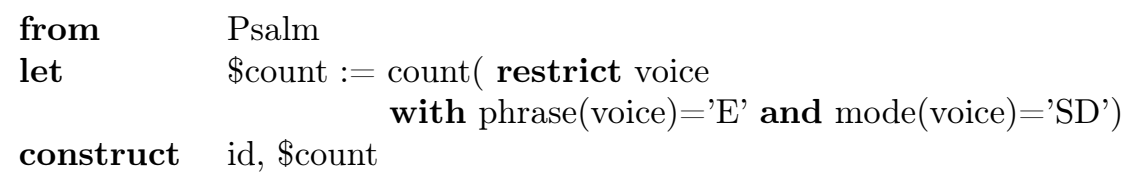

In summary, the language offers a mechanism to search and transform music representations, combine them and produce new scalar or temporal information. The model allows to manipulate scalar values representing global information on a music piece, and temporal function that give the detail of music events occurrences at each instant. These core operations must be completed with functions that can be natively integrated in language expressions. Finally, the derivation mechanism briefly introduced above provides a simple way to create new time series from sequence of "local views" of one or several time series.

\section{Conclusion and ongoing work}

The model presented here was designed and developped in the context of the Neuma project, which aims at designing and implementing a digital library devoted to the preservation and dissemination of symbolic music content (scores). The score modeling developed in the present paper aims at defining a sound query interface to the NeumA services. By adopting from the beginning an algebraic approach to the management of time series data sets, we directly enable an expressive and stable language that avoids a case-by-case definition of a query language based on the introduction of ad-hoc functions subject to constant evolution. We believe that this constitutes a sound basis for the development of applications that can rely on an expressive and efficient data management.

The query language is currently under development. A preliminary form is being used with the monodic collections already supported by Neuma. The full implementation will serve to handle polyphonic collections that must be 
integrated in a near future. A side effect of a query language design based on limited algebra is the development of access paths to efficiently support common searches on large music collections. Our short-term roadmap also includes an investigation of indexing structures apt at retrieving patterns in large collections.

Acknowledgments. This work is partially supported by the French ANR NEUMA project, http://neuma.irpmf-cnrs.fr. The authors would like to thank Virginie Thion-Goasdoue and David Gross-Amblard.

\section{References}

1. Allan H., Mï $\measuredangle \frac{1}{2}$ llensiefen D. and Wiggins,G.A.: Methodological Considerations in Studies of Musical Similarity, Proc. Intl. Society for Music Information Retrieval (ISMIR) (2007)

2. Anglade, A. and Dixon, S.: Characterisation of Harmony with Inductive Logic Programming, Proc. Intl. Society for Music Information Retrieval (ISMIR) (2008)

3. Anglade, A. and Dixon, S.: Towards Logic-based Representations of Musical Harmony for Classification Retrieval and Knowledge Discovery, MML (2008)

4. Berman, T., Downie, J. and Berman, B.: Beyond Error Tolerance: Finding Thematic Similarities in Music Digital Libraries,Proc. European. Conf. on Digital Libraries, pp 463-466 (2006) pages $=463-466$

5. Berndt, D. and Clifford, J.: Using dynamic time warping to find patterns in time series. AAAI Workshop on Knowledge Discovery in Databases, pp. 229-248 (1994)

6. Brockwell, P.J. and Davis, R.: Introduction to Time Series and forecasting, Springer-Verlag, (1996)

7. Cameron, J., Downie, J.S and Ehmann, A.F.: Human Similarity Judgments: Implications for the Design of Formal Evaluations, Proc. Intl. Society for Music Information Retrieval (ISMIR) (2007)

8. Capela, A., Rebelo, A. and Guedes, C.: Integrated recognition system for music scores, Proc. of the 2008 Internation Computer Music Conferences (2008)

9. Downie, J. and Nelson, M.: Evaluation of a simple and effective music information retrieval method, Proc. ACM Symp. on Information Retrieval (2000)

10. Downie, JS.: Music Information Retrieval, Annual review of Information Science and Technology vol. 37, pp. 295-340 (2003)

11. Ganseman J., Scheunders P. and D'haes, W.: Using XQuery on MusicXML Databases for Musicological Analysis, Proc. Intl. Society for Music Information Retrieval (ISMIR) (2008)

12. Good, M.: MusicXML in practice: issues in translation and analysis, Proc. 1st Internationl Conference on Musical Applications Using XML, pp. 47-54 (2002)

13. Haus, G., Longari, M. and Pollstri,E.: A Score-Driven Approach to Music Information Retrieval, Journal of American Society for Information Science and Technology, vol 55, pp. 1045-1052 (2004)

14. Huron, D.: Music information processing using the HumDrum toolkit: Concepts, examples and lessons, Computer Music Journal 26, 11-26 (2002)

15. Keogh, E.J and Ratanamahatana, C.A. : Exact Indexing of Dynamic Time Warping, Knowl. Inf. Syst. vol. 7, number 3, pp. 358-386 (2003)

16. Knopke, I. : The Perlhumdrum and Perllilypond Toolkits for Symbolic Music Information Retrieval, Proc. Intl. Society for Music Information Retrieval (ISMIR) (2008) 
17. Lee J.Y. and Elmasri, R.: An EER-Based Conceptual Model and Query Language for Time-Series Data, Proc. Intl.Conf. on Conceptual Modeling, pp. 21-34 (1998)

18. Lerner, A., Shasha, D.: AQuery : Query language for ordered data, optimization techniques and experiments, Proc. of the 29th VLDB Conference, Berlin, Germany (2003)

19. Muller, M.: Information Retrieval for Music and Motion, Springer (2004)

20. Rabiner, L., Rosenberg, A. and Levinson, S.: Considerations in dynamic time warping algorithms for discrete word recognition, IEEE Trans. Acoustics, Speech and Signal Proc, vol ASSP-26 pp. 575-582 (1978)

21. Sakoe, H. and Chiba, S. : Dynamic programming algorithm optimization for spoken word recognition, IEEE Trans. Acoustics, Speech and Signal Proc, vol ASSP-26 pp. 43-49 (1978)

22. Sapp, CS. : Online Database of Scores in the Humdrum File Format, Proc. Intl. Society for Music Information Retrieval (ISMIR) (2005)

23. Typke, R., Wiering, F. and Veltkamp, R.C.: A Survey Of Music Information Retrieval Systems, Proc. Intl. Society for Music Information Retrieval (ISMIR) (2005)

24. Viglianti, R.: MusicXML : An XML based approach to automatic musicological analysis, Conference Abstracts of the Digital Humanities (2007)

25. Zhu, Y. and Shasha, D.: Warping Indexes with Envelope Transforms for Query by Humming, pp. 181-192, Proc. ACM SIGMOD Symp. on the Management of Data (2003)

26. Mutopia, http://www.mutopiaproject.org

27. Melodicmatch, http://www.melodicmatch.com

28. Musipedia, http://www.musipedia.org

29. Wikifonia, http://www.wikifonia.org

30. Neuma, http://neuma.irpmf-cnrs.fr 